\section{The authors respond to "Opioid agonist dosage adequacy from clinical and patient perspectives: further considerations"}

We appreciate the comments by Trujols and colleagues ${ }^{1}$ on our CMAJ article. ${ }^{2}$ We wish to highlight that the thresholds used to dichotomize the prescribed dosage for opioid agonist treatment in our study were informed by 3 national clinical guidelines on opioid management in the United States, ${ }^{3}$ Canada $^{4}$ and the United Kingdom, ${ }^{5}$ and also by a guideline proposed by the World Health Organization. ${ }^{6}$ These recommendations are largely based on 2 systematic reviews illustrating that methadone dosages of at least $60 \mathrm{mg} / \mathrm{d}$ are more effective at retaining individuals in treatment and reducing illicit opioid use compared with lower doses. ${ }^{7,8}$ Similarly, most guidelines recommend offering dosages of at least $16 \mathrm{mg} / \mathrm{d}$ for buprenorphine, ${ }^{3-6}$ in line with findings from a systematic review. ${ }^{9}$

Altogether, the thresholds that we used in our study were based on the best available evidence around optimal effective dosages at the population level. In addition, we explored potential heterogeneity across binary (i.e., high/low) categories of opioid agonist dosage, informed by a priori-specified thresholds, with high/ moderate/low categories based on tertiles observed in our sample, as a subanalysis.

As Trujols and colleagues point out, ${ }^{1}$ the range of effective dosages is broad. For this reason, we did not limit our assessment of opioid agonist treatment adequacy to only a measure of dosage but also considered patients' perspectives. The Opiate Dosage Adequacy Scale (ODAS) mentioned by Trujols and colleagues can be a valuable clinical tool to help inform dosing decisions around opioid agonist treatment in practice. Whether clinicians ask patients about their self-perceived adequacy of dosage, use the ODAS or other scales to optimize the dosage, it is important that patients are involved as active partners in their treatment and care for opioid use.

As also pointed out by Trujols and colleagues, flexible dosing strategies, in which the opioid agonist treatment dosage is adjusted to individual need rather than following predetermined fixed-dose regimens, have been found to be effective in practice. ${ }^{10}$

We believe that the average therapeutic range and the dosing strategy are 2 distinct, albeit equally important, aspects of opioid agonist treatment. In their meta-analysis examining the relative role of methadone dose (60 or more v. less than $60 \mathrm{mg} / \mathrm{d}$, defined as high/low) and dosing strategy (flexible/fixed), Bao and colleagues elegantly showed that retention in treatment was greater at high doses across both dosing strategies. ${ }^{10}$ Conversely, a greater retention in treatment was also observed with flexibledose relative to fixed-dose strategies across high/low doses. As the authors carefully pointed out, "it is likely that retention will be greatest when the dosing strategy is flexible and doses are relatively high." ${ }^{10}$

We agree with Trujols and colleagues that the optimal dosage for opioid agonist treatment should be informed by average therapeutic doses at the population level and tailored to individual patients' needs and preferences.

\section{Andreea A. Artenie MSc}

PhD candidate, School of Public Health, Université de Montréal, Montréal, Que.

Julie Bruneau MD MSc

Professor, Department of Family and Emergency Medicine, Université de Montréal, Montréal, Que.
Cite as: CMAJ 2019 September 30; 191:E1085. doi: 10.1503/cmaj.73208

\section{References}

1. Trujols J, Campins JM, Ribalta E. Opioid agonist dosage adequacy from clinical and patient perspectives: further considerations [letter]. CMAJ 2019;191:E1084

2. Artenie AA, Minoyan N, Jacka B, et al. Opioid agonist treatment dosage and patient-perceived dosage adequacy, and risk of hepatitis $C$ infection among people who inject drugs. CMAJ 2019;191:E462-8.

3. Kampman K, Jarvis M. American Society of Addiction Medicine (ASAM) national practice guideline for the use of medications in the treatment of addiction involving opioid use. J Addict Med 2015;9:358-67.

4. Bruneau J, Ahamad K, Goyer ME, et al.; CIHR Canadian Research Initiative in Substance Misuse. Management of opioid use disorders: a national clinical practice guideline. CMAJ 2018; 190:E247-57.

5. Clinical Guidelines on Drug Misuse and Dependence Update 2017 Independent Expert Working Group. Drug misuse and dependence: UK guidelines on clinical management. London (UK): Department of Health; 2017. Available: https:// assets.publishing.service.gov.uk/government/ uploads/system/uploads/attachment_data/file /673978/clinical_guidelines_2017.pdf (accessed 2018 June 5).

6. Guidelines for the psychosocially assisted pharmacological treatment of opioid dependence. Geneva: World Health Organization; 2009. Available: www. who.int/substance_abuse/publications/opioid _dependence_guidelines.pdf (accessed 2018 June 4).

7. Faggiano $F$, Vigna-Taglianti $F$, Versino $E$, et al. Methadone maintenance at different dosages for opioid dependence. Cochrane Database Syst Rev 2003;(3):CD002208.

8. Fareed A, Casarella J, Amar R, et al. Methadone maintenance dosing guideline for opioid dependence, a literature review. J Addict Dis 2010;29:1-14.

9. Mattick RP, Breen C, Kimber J, et al. Buprenorphine maintenance versus placebo or methadone maintenance for opioid dependence. Cochrane Database Syst Rev 2014;(2):CD002207.

10. Bao YP, Liu ZM, Epstein DH, et al. A meta-analysis of retention in methadone maintenance by dose and dosing strategy. Am J Drug Alcohol Abuse 2009;35:28-33.

Competing interests: None declared. 